\title{
Micro Gravity Experiment and 3 Dimensional Dynamic Analysis of Tethered Sampler
}

\author{
By Takeo WATANABE ${ }^{1)}$, Hironori A. FUJII ${ }^{2)}$, Hirohisa KOJIMA ${ }^{1)}$, \\ Hiroshi TAKIKAWA ${ }^{1)}$, Masakazu YUKIZANE ${ }^{1)}$ and Kazuki ITO ${ }^{1)}$ \\ ${ }^{1)}$ Department of Aerospace Engineering, Tokyo Metropolitan University, Tokyo, Japan \\ ${ }^{2)}$ Department of Aerospace Engineering, Kanagawa Institute of Technology, Kanagawa, Japan
}

(Received April 25th, 2008)

\begin{abstract}
As a new sampling technique, a "Harpoon \& Penetrator" method by a tethered sampler is planned instead of "Touch and Go" method for the next sample return project of small celestial bodies explorations. This paper reports about the results of micro gravity experiments by using parabolic flights of the airplane in order to analyze 3-dimensional dynamic behavior of the tethered rigid body. Objectives of the experiments are demonstration of feasibility of the present sampling method and observation of dynamic characteristics in micro gravitational environments. The extraction / catching experiments and the launch / deployment experiments are explained in this paper. These results contribute to the feasibility study of the tethered sampler and will help to understand the characteristics and to make trade off of the tethered sampling methods.
\end{abstract}

Key Words: Tether, Sample Return, Micro Gravity Experiment,

\section{Introduction}

In the Muses-C (Hayabusa) mission, a "Touch \& Go method" sequence was employed to collect the asteroid samples. This method was effective to collect samples from the asteroid with unknown surface conditions such as flatness and hardness but it was able to catch only by a few grams of the total mass. In the next sample return project of small celestial bodies explorations, ${ }^{1)}$ a new sampling technique, a "Harpoon \& Penetrator" method by the tethered sampler is planned instead of a "Touch and Go" method. In the sample return of the post-Hayabusa mission concept, several ten gram soils in the depth from 20 to $30 \mathrm{~cm}$ in layer are desired to be caught and returned (Fig.1).

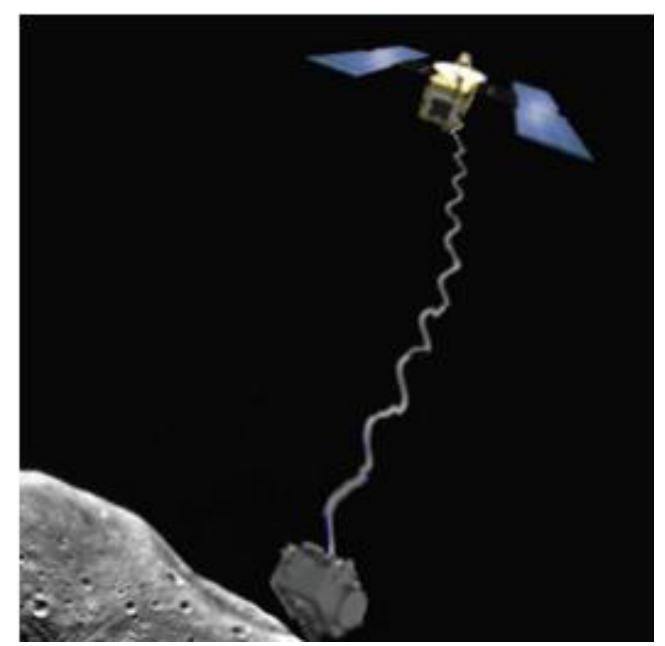

Fig. 1. Asteroid Explorer and Tethered Sampler.
We found the principle of the application of tether technology to Comet/Asteroid Sample Return in the Tethers in Space Handbook. ${ }^{2)}$ In the application, tethered penetrators are launched from a spacecraft during its rendezvous with the target body. With the rapid progress of the guidance navigation, and control technology in recent years, non-landed surface sampling missions are under investigation. In order to keep a high survivability of spacecraft, those missions plan to equip tethered penetrators or tethered robots equipped with the sampling devices. ${ }^{3-5)}$ Following up the Hayabusa mission, Japanese working group has started a feasibility study and discussion of several types of non-landing sampling methods. Some of them include application of tether technology. From the viewpoint of survivability, the sampling device should be designed to be as simple as possible. In addition, the equipment must have robustness and reliability. ${ }^{6-8)}$ Deployment, retrieval or control problems of tether systems are widely studied due to their scientific/ engineering interests. Especially experimental evaluations of hardware are important in order to understand dynamic characteristics or development of devices in a micro gravity environment ${ }^{8-11)}$.

This paper reports on the results of micro gravity experiments by parabolic flights of the airplane. In the present Ground-based Research Program, parabolic flight experiments have been carried out in order to analyze the three-dimensional dynamic behavior of the tethered rigid body. ${ }^{8)}$ The objectives of the experiments are demonstration of feasibility of the present sampling method and the observation of the dynamic characteristics in micro gravitational environments. The extraction/catching experiments and the launch/deployment experiments have been carried out. These results contribute to the feasibility study of the tethered sampler and will help to understand the dynamic characteristic under micro gravitational 
condition of tether system and to make trade offs of the tethered sampling methods.

\section{Tethered Sampler}

The sampling sequence of the tethered sampler consists of (1) launch phase, (2) penetration and extraction phase, (3) catching phase, and (4) capsulation phase as shown in the schematic views in Fig.2. The explorer does not need to land or to contact the target asteroids in this strategy.

The main advantages of the tethered sampler method can be described as follows: 1) Safety sampling process from high altitude $(15 \sim 20 \mathrm{~m})$ can be operated in order to avoid the risk of collision of the explorer with the surface. 2) The amount of samples expected to be obtained is larger than that of the previous method, and the order of samples towards depth direction can be kept. Although there are such advantages, the tethered sampler has complex dynamic characteristics. In particular, the characteristics of the launch or extraction phases should be analyzed numerically and experimentally.

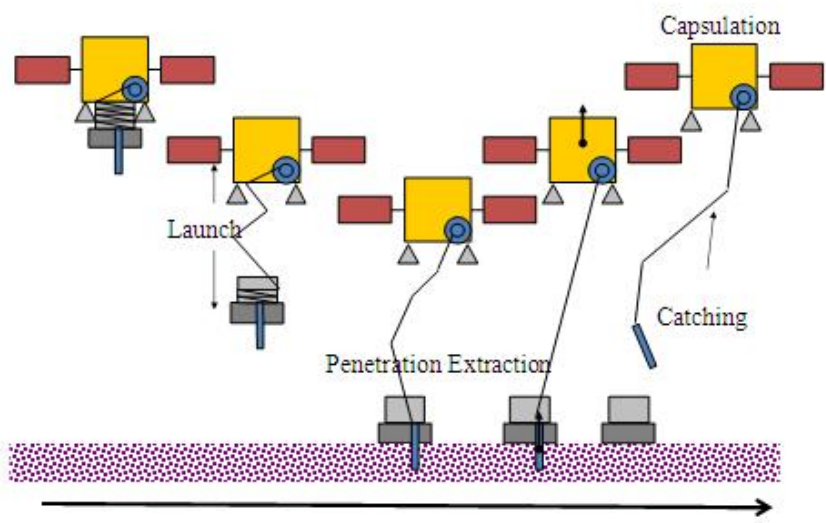

Fig. 2. Schematic of Sampling Sequence.

\section{Experiment Setup}

Dynamics of the tethered sampler can be modeled by the motions of a flexible tether and tethered rigid body. In order to analyze three-dimensional dynamic behavior of the tethered rigid body, parabolic flight experiments have been carried out in the summer and the winter of 2007. In the experiment, shooting motion of the launch phase and pull-out motions of the extraction phase have been studied experimentally in the airplane (Mu-300).

\subsection{Mu-300}

The parabolic flight of Mu-300 airplane can provide micro gravity conditions for approximately 20 seconds. The opportunity of the micro gravity experiment has been supported by Japan Space Forum and Diamond Air Service Inc. The Mu-300 mounts measurement devices and data logger, thus, synchronized experimental data can be recorded.

\subsection{Gyro Sensor}

The body of the airplane is rotated approximately -90 degrees in pitch at each parabolic flight. The pitch motion can affect the dynamics of the instruments in the experimental space. The Gyro sensors "CRS-03" produced by Silicon Sensing Systems Japan Ltd. were selected to measure the rotational motion (Fig. 3). Measured pitch rates were recorded with other data synchronously.

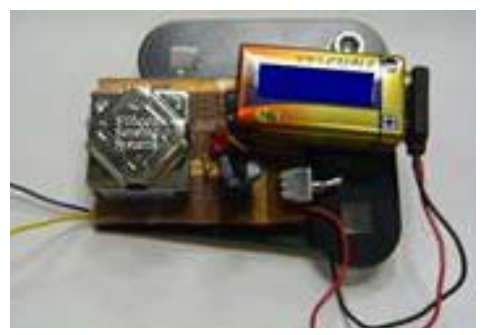

Fig. 3. CRS-03 Gyro sensor.

\subsection{Cameras}

In order to obtain three-dimensional behavior of the target object, ten cameras were set up internally including a panorama view. These cameras were synchronized by light before and after each experiment, and we obtained the coordinate data of three dimensions by image processing. The scheme is explained in the next section.

\subsection{DLT method}

Direct Liner Transformation (DLT) method was employed in order to process the three-dimensional motions of the target in microgravity experimental space.

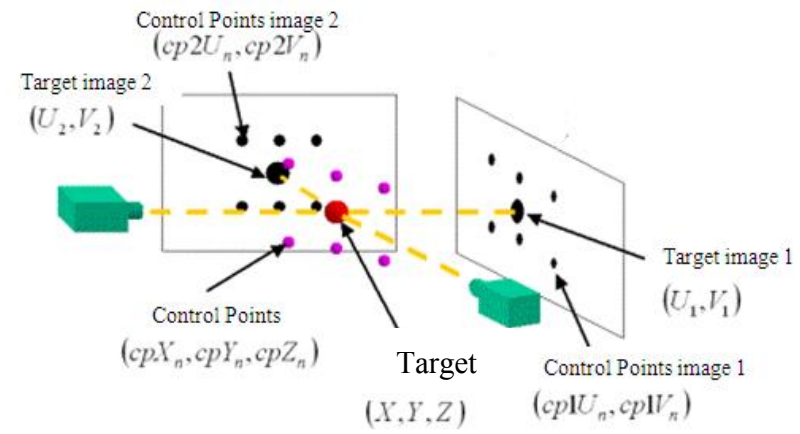

Fig. 4. DLT Method and Control points.

Fig. 4 shows the schematic expression of DLT method. The DLT method is one of the image processing methods in order to obtain the optional coordinates using two or more video cameras. In general, cameras are required to obtain the three-dimensional coordinates from the image taken from two different directions. However, it is not easy to acquire precise values. The DLT method has the advantage of avoiding this problem by using control points defined in the predefined three-dimensional coordinates.

\section{Launch / Deployment Phase}

Safe and reliable deployment of tether is required in the launch phase of the sampling sequence. A z-folded type tape tether deployment system is selected in the system. The 
deployment system was designed to be simple to keep enough reliability in the high-speed deployment. The deployment system has originally been developed for the sounding rocket experiment ${ }^{12)}$ to deploy an electro conductive tape tether with high speed.

\subsection{System block diagram and experiment setup}

Fig. 5 shows the experimental setup and system block diagram for the launch / deployment phase. The tethered sampler is deployed by a spring mechanism. The launcher has a table rotated by a DC torque motor and a timing belt in order to provide spin or disturbance for the launched sampler. Although the sampler model is designed as cylindrical shape with diameter of $200 \mathrm{~mm}$, there are several miss-alignments between the launch axis and the principal axis of inertia. The tether is stored in the box mounted on the sampler (Fig.6). The specifications are listed in Table 1.

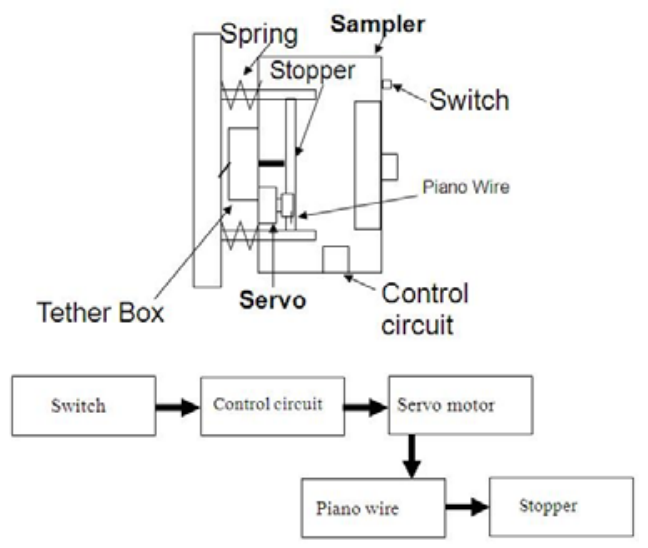

Fig. 5. Setup of the Launch / Deployment Phase Experiment and block diagram.

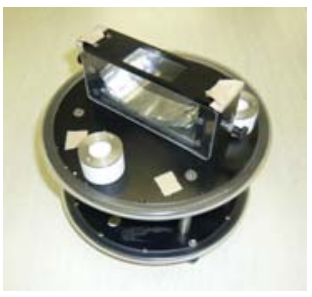

(a)

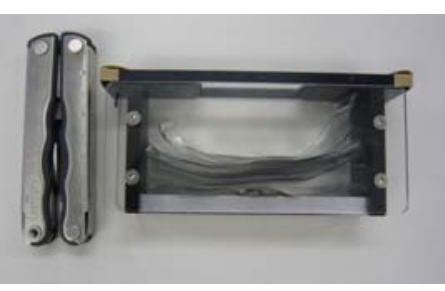

(b)
Fig. 6. Sampler model (a) and tether storage box (b).

Table 1. Specifications of Launch phase experiment

\begin{tabular}{|c|c|}
\hline Tether length & $2.15[\mathrm{~m}]$ \\
\hline Foldaway pitch & $100[\mathrm{~mm}]$ \\
\hline Tether width & $20[\mathrm{~mm}]$ \\
\hline Mass of sampler & $1.2[\mathrm{~kg}]$ \\
\hline Initial velocity & $0.5[\mathrm{~m} / \mathrm{s}]$ \\
\hline
\end{tabular}

\subsection{Experiential results}

Fig. 7 shows one result of launch / deployment phase experiments. The deployed sampler flew from the launcher, and deployment behavior of folded tether was observed. Due to the simple mechanism (without rotational elements), the foldaway tether deployer is expected to have less dynamic disturbance effect on the spacecraft body during the rapid deployment. The entire tether has been safely deployed even in the presence of disturbances at the launch phase, or even in the case of an unbalanced sampler with additional weights.

From these results, we can say that the tethered sampler can be launched with enough reliability of tether deployment.
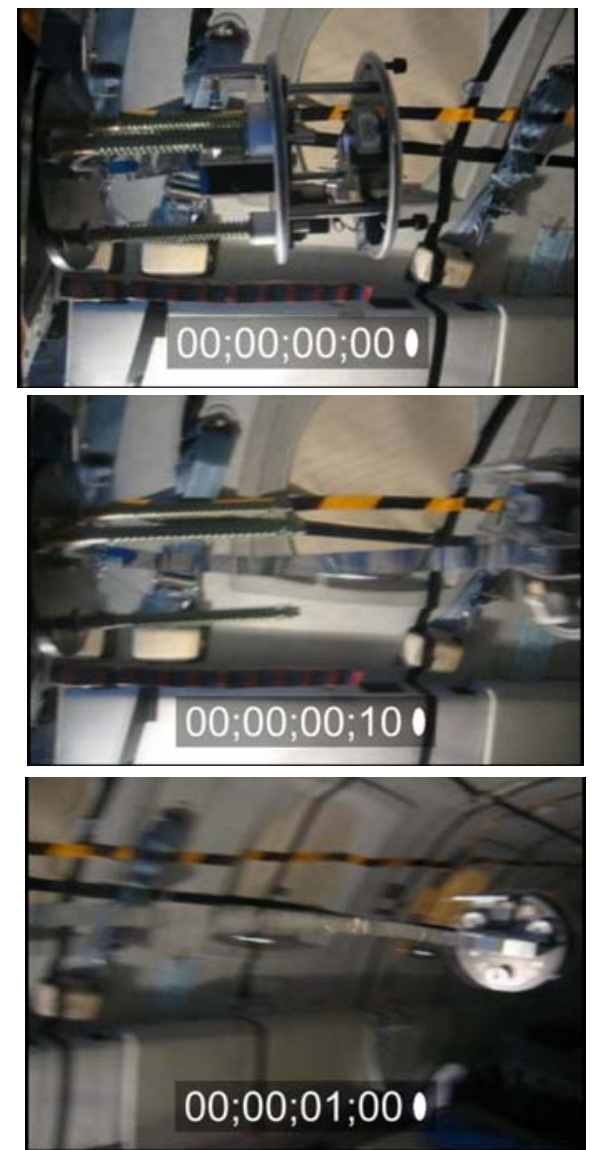

Fig 7. Launch / Deployment phase experiment (30 frames per second).

\section{Extraction / Catching Phase}

The dynamic behaviors of the tethered rigid bodies were observed by filming, and we have obtained complex phenomenon of the extraction / catching phase.

Trajectory and attitude motion of tethered corer are plotted by processing the image data by the DLT method. Distributions of the arrival points are analyzed to evaluate the safety of catching phase under various extracting conditions.

\subsection{System model and experimental setup}

Coordinates and conditions of the extracted sampling system are shown in Fig.8. The condition of extraction depends on the state of penetration, and in this study it is defined by penetration attitude $\theta, \psi$, and tether tension angle $\phi$.

Fig. 9 shows the system block diagram of the extraction / catching phase experiment. The experiment setup consists of the holder, tape tether, corer, extraction device, PC, video cameras, sensors, and data logger. The tether tension is generated by a pressed spring, and a load cell measures the tension on the extracting device. The extraction device pulls the tether to extract the sampler set under the optional boundary conditions. 


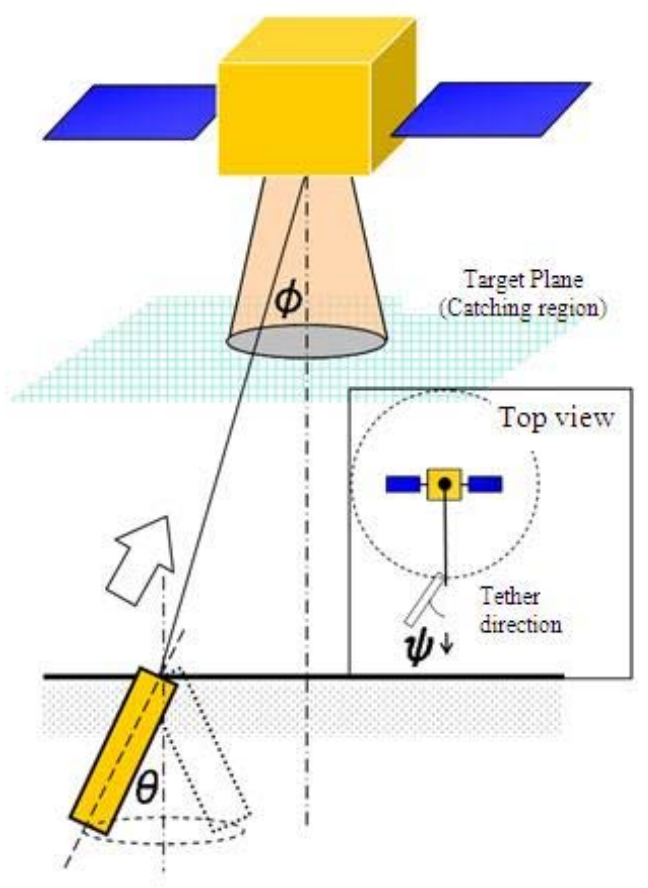

Fig. 8. System model of the Extraction / Catching phase.

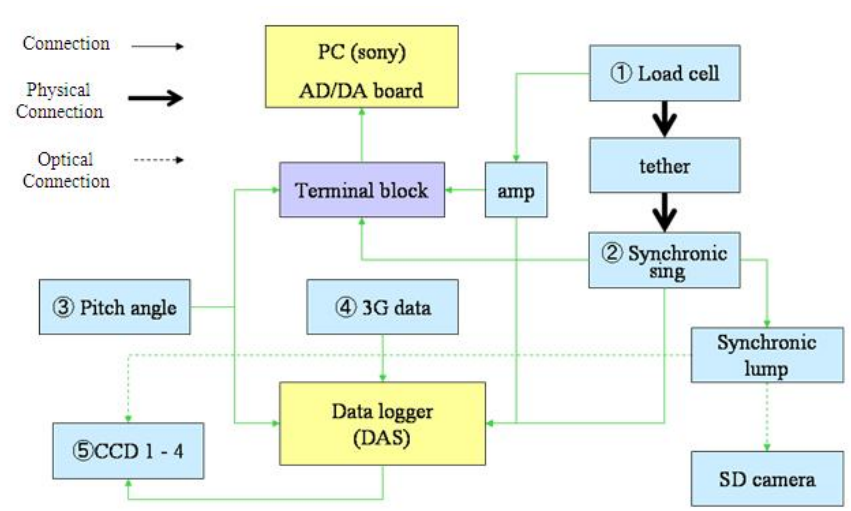

Fig 9. System block diagram of the Extraction / Catching phase experiment.

The holder and the extraction device are located on the nose side of the plane, and on the tale side, respectively, as shown in Fig. 10. Specifications of the experimental setup are listed in Table 2.

\subsection{Results and discussions}

One of the typical test results obtained in the parabolic flight is shown in Fig 11. Flight acceleration levels of the parabolic flight have been measured relative to the fixed reference frame of the aircraft.

Three axis acceleration levels are shown in Fig.11(a). The pitch rate and the tension data are also measured and those data are synchronized with the trigger signal of the micro switch activated at the time of extraction.

The pitch rate of the parabolic flight was measured approximately as $-0.8[\mathrm{rad} / \mathrm{sec}]$, and this motion affects the trajectory of the objects in the plane.

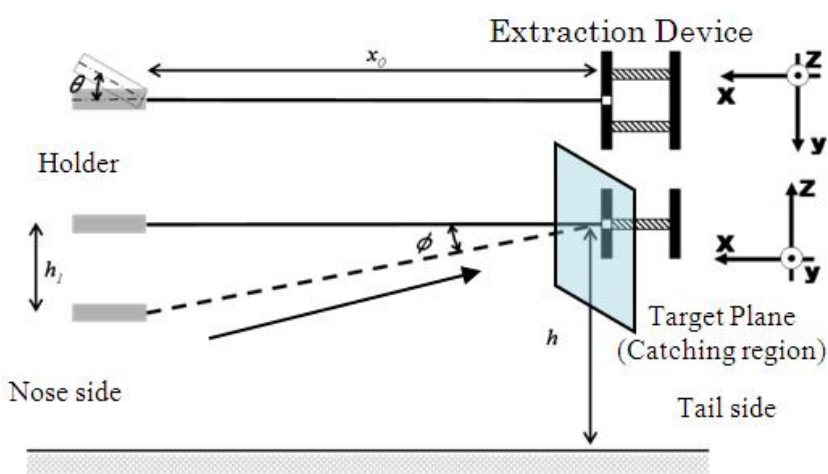

Fig. 10. Experiment setup.

Table 2. Specifications

\begin{tabular}{|l|c|c|}
\hline $\mathrm{x}_{0}$ & 2.5 & $\mathrm{~m}$ \\
\hline $\mathrm{h}$ & 0.69 & $\mathrm{~m}$ \\
\hline $\mathrm{h}_{1}$ & 0.3 & $\mathrm{~m}$ \\
\hline$\varphi$ & 6.8 & $\mathrm{deg}$ \\
\hline Corer length: $\mathrm{l}$ & 70 & $\mathrm{~mm}$ \\
\hline Corer Diameter: d & 20 & $\mathrm{~mm}$ \\
\hline Corer Mass: m & 0.13 & $\mathrm{~kg}$ \\
\hline Moment of Inertia Jx & $8.4 \times 10^{-5}$ & $\mathrm{~kg} \mathrm{~m}^{2}$ \\
\hline Moment of Inertia Jy & $8.4 \times 10^{-5}$ & $\mathrm{~kg} \mathrm{~m}^{2}$ \\
\hline Moment of Inertia Jz & $7.0 \times 10^{-6}$ & $\mathrm{~kg} \mathrm{~m}^{2}$ \\
\hline
\end{tabular}

The pitch rate data was used to correct the results obtained by the camera mounted in the plane. The recorded tether tension was similar to the one estimated before the experiments. Maximum tensions were $25 \sim 30[\mathrm{~N}]$, which took place around 0.02 0.03 seconds after the extraction.

One typical dynamic behavior of the extracted corer and tether is shown in Fig. 12, where the corer was extracted under the initial conditions: $\phi: 7[\mathrm{deg}], \theta: 30[\mathrm{deg}]$, and $\psi: 90[\mathrm{deg}]$. The corer flew from the holder to the extraction device with the initial velocity given by the extracting tension. The initial velocity of the corer after extraction was approximately $1.5[\mathrm{~m} / \mathrm{s}]$, and the corer arrived at about 50 [cm] below the extraction device two seconds after the extraction.

In the early stage of the extraction phase, the rotation of the corer was observed. The rotational axis of the corer depended on the tether tension vector and the holding vector of the holder. This implies that the rotational motion of the corer will depend on condition of penetration.

During the flight the rotating corer seems to wrap the slack tether, and the kinetic energy of rotation is supposed to be damped out by the effect of tether motion and air drag. 


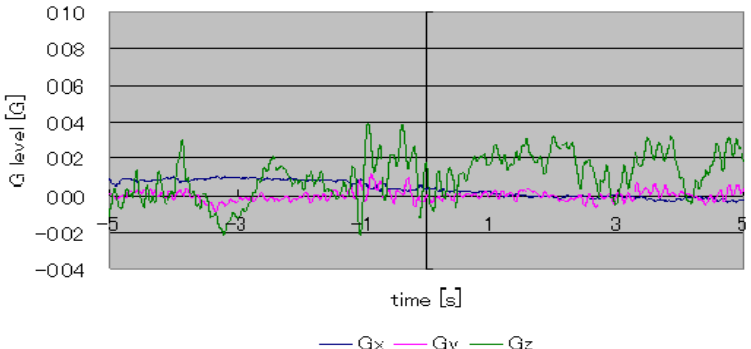

(a)

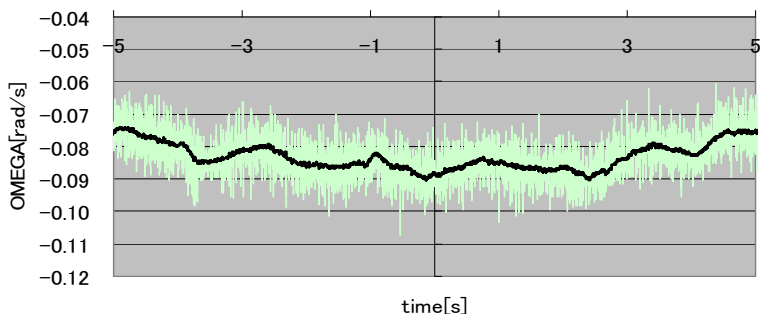

(b)

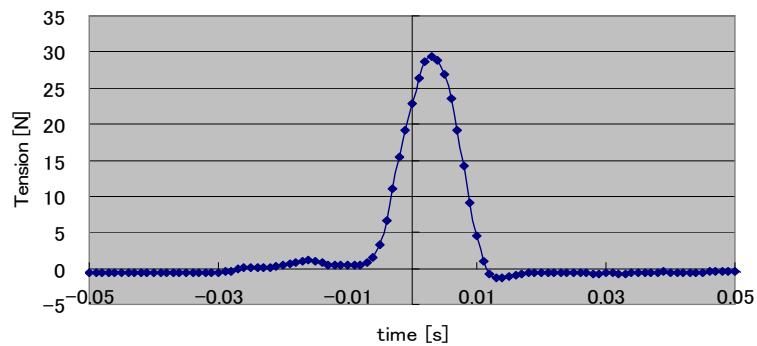

(c)

Fig .11. Typical test results; three-axis acceleration (a), Pitch rate of the plane (b), and tension at the extraction device(c).

It is shown in Fig.12 that the arrival points of the corer at the extraction device side shifted downwards due to the effects of the pitch rotation of the airplane, or acceleration of $\mathrm{z}$ direction.

In order to study the dependence of the arrival positions on the extraction conditions, experiments under various extraction conditions were carried out. Parameters of the experiment are listed in Table 3. Distributions of the arrival points are shown in Fig. 13. The number in Fig. 13 corresponds to the condition number in Table 3.

To express the arrival positions of the object, z-y plane is defined at the extraction device. Unit of the grid is $\mathrm{cm}$, and the origin of the z-y plane is set on the extraction point. The dependence of the arrival positions on the extraction conditions was evaluated by grouping the arrival points at the z-y plane. The results showed that the corer flew towards the right side and arrived at the device in the cases of the holder angle $\theta$ of $30[\mathrm{deg}]$. This is because the initial velocity was induced towards the right side by the tether tension vector and penetration attitude.

Although all the parabolic flight were not always the same, the results showed the tendency of trajectories of the extracted corer.

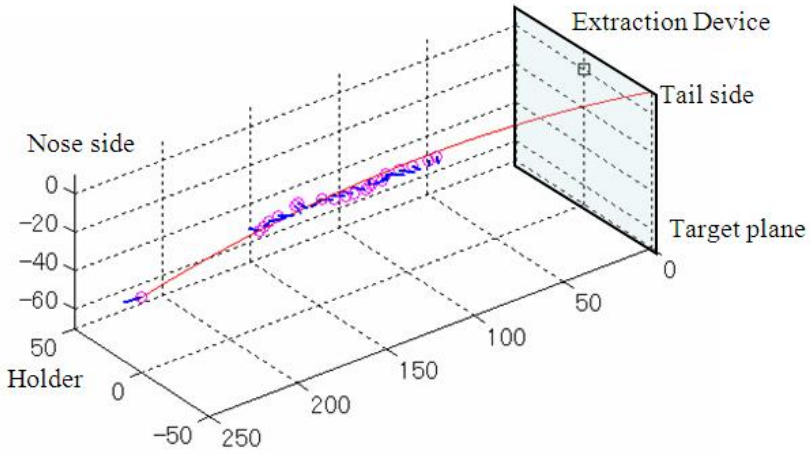

(a)
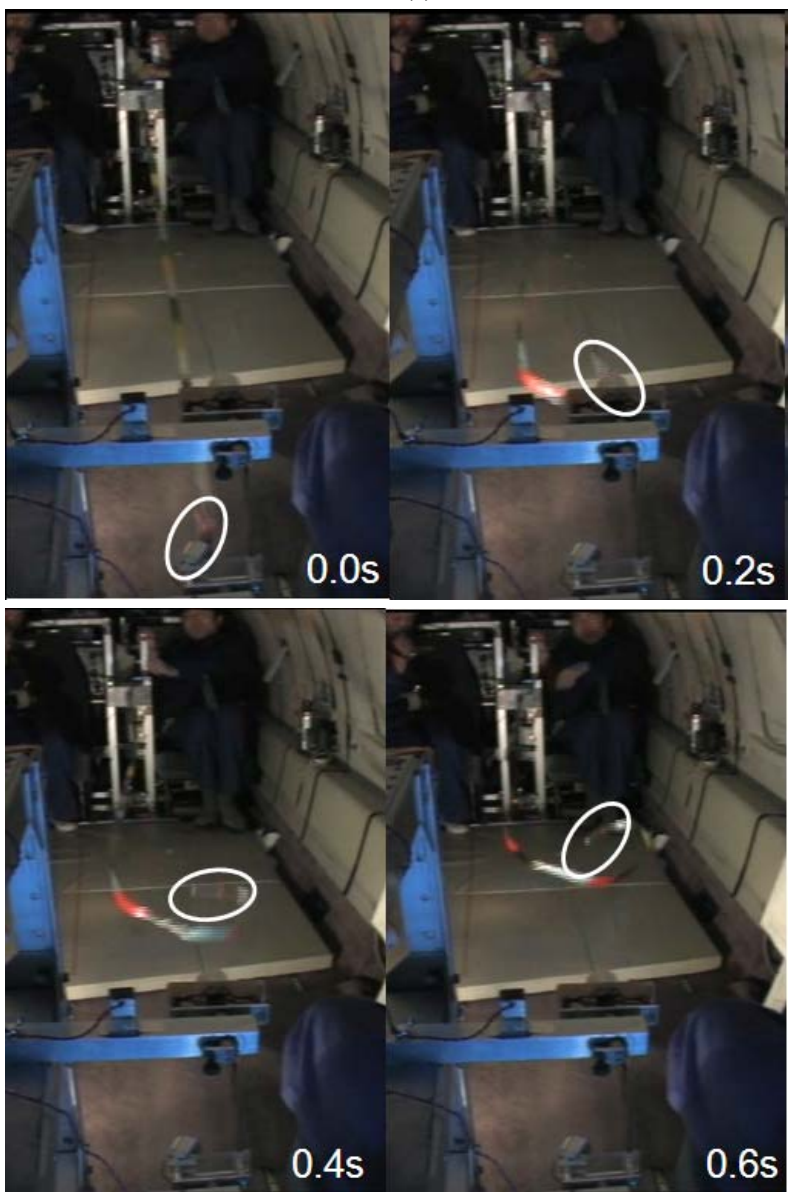

(b)

Fig. 12. Behavior of the extracted tethered sampler (a), and the photographs (b).

\section{Conclusions}

A concept of tethered sampler is presented. Feasibility of the tethered sampler has been inspected experimentally in this paper. Launch/ deployment phase and extraction/ catching phase of the tethered sampling sequence were operated in three-dimensional micro gravity environment using parabolic flight. The tethered sampler is deployed by spring force. The launch tests have shown feasibility of the tethered sampler deployment.

Utilizing the results presented in this paper, we will design a simple deployment / sampling return system to keep enough reliability in the high-speed deployment /retrieval. 
Table 3. Parameters of Extraction / Catching Phase experiment

\begin{tabular}{cccccc}
\hline \hline No. & $\begin{array}{c}\text { Width } \\
{[\mathrm{mm}]}\end{array}$ & $\begin{array}{c}\phi \\
{[\mathrm{deg}]}\end{array}$ & $\begin{array}{c}\theta \\
{[\mathrm{deg}]}\end{array}$ & $\begin{array}{c}\Psi \\
{[\mathrm{deg}]}\end{array}$ & $\begin{array}{c}\mathrm{n} \\
{[\mathrm{roll} / \mathrm{m}]}\end{array}$ \\
\hline \hline 01 & 20 & 0 & 0 & 0 & 0 \\
$\# 02$ & 20 & 0 & 0 & 0 & 0 \\
$\# 03$ & 20 & 0 & 0 & 0 & 1.2 \\
$\# 04$ & 20 & 0 & 0 & 0 & 1.2 \\
$\# 05$ & 20 & 7 & 30 & 90 & 0 \\
$\# 06$ & 20 & 7 & 30 & 90 & 0 \\
$\# 07$ & 20 & 7 & 30 & 90 & 1.2 \\
$\# 08$ & 20 & 7 & 30 & 90 & 1.2 \\
$\# 09$ & 20 & 0 & 30 & 0 & 0 \\
$\# 10$ & 20 & 0 & 30 & 0 & 0 \\
$\# 11$ & 20 & 0 & 30 & 0 & 1.2 \\
$\# 12$ & 20 & 0 & 30 & 0 & 1.2 \\
$\# 13$ & 20 & 7 & 0 & 0 & 0 \\
$\# 14$ & 20 & 7 & 0 & 0 & 0 \\
$\# 15$ & 20 & 7 & 0 & 0 & 1.2 \\
$\# 16$ & 20 & 7 & 0 & 0 & 1.2 \\
$\# 17$ & 20 & 7 & 0 & 0 & 1.2 \\
$\# 18$ & 20 & 0 & 30 & 0 & 1.2 \\
$\# 19$ & 20 & 0 & 30 & 0 & 1.2 \\
$\# 20$ & 20 & 0 & 30 & 0 & 1.2 \\
\hline & & & & &
\end{tabular}

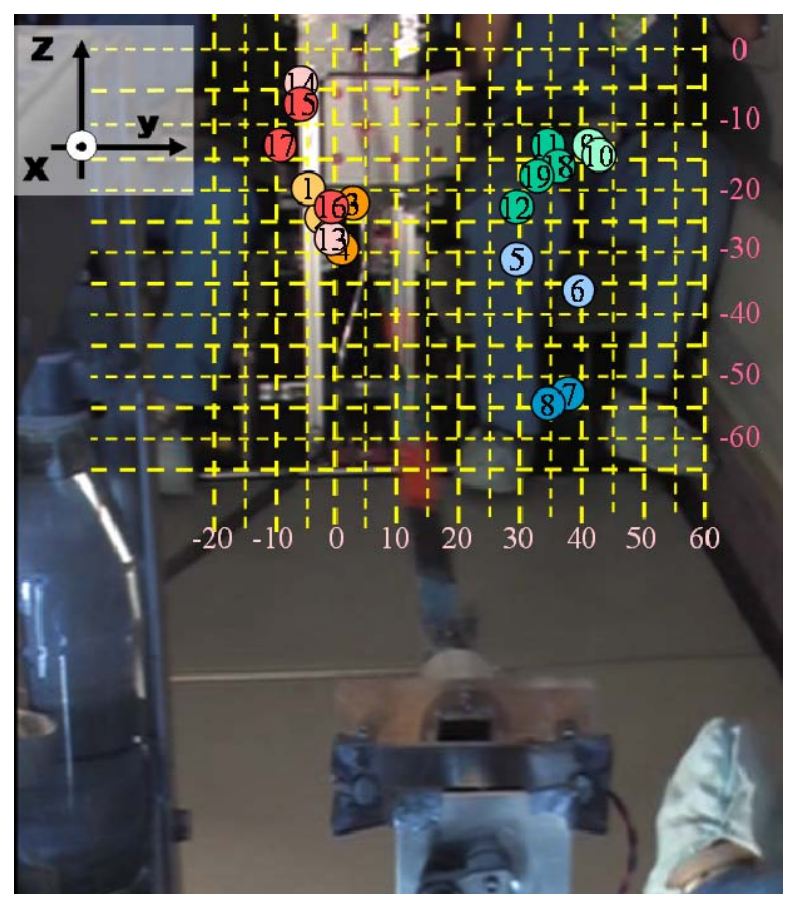

Fig. 13. Arrival positions of the extracted corer.

\section{Acknowledgements}

This research was supported by the "Ground-based Research Program for Space Utilization" promoted by Japan Space Forum, and supported by JAXA.

\section{References}

1) Fujii, H. A., Takikawa, H., Yukizane, M., amd Watanabe, T. Study of Sampling Method of a Rigid Sampler Connected by Tether, Paper 2006-k-30, Proceedings of 25th International Symposium of Space Technology and Science 2006, pp. 1083-1087.

2) Tether in space Handbook, Prepared for NASA Marshall Space Flight Center,Third Edition December 1997, pp83-84

3) Bartlett, P. W., Basso, B., Paulsen, G., Gorevan, S. and Yachbes, I.: Asteroid \& Comet Sureface Sampling Methods from Non-Landed Spacecraft, Workshop on Spacecraft Reconnaissance of Asteroid and Comet Interior, California, 2006.

4) Behar, A., (2003) JPL TRS http://hdl.handle.net/2014/7305

5) Yoshida, K.: Critical Motion Analysis on Touch-Down Sampling of MUSES-C, Proceedings of 12th Workshop on Astrodynamics and Flight Mechanics, 2002.

6) Yano, H., Noguchi, T., Matsunaga, S., Mori, O., Habu, H., Hasegawa, S., Kato, K., Kubota, S., Miura Y., Nakaya, K., and Nozoe, K.: Development of the Sampling Device for Undifferentiated Asteroid Surface and Sub-Surface Materials, Proceedings of 25th International Symposium of Space Technology and Science, 2006-k-29, 2006, pp. 1071-1075.

7) Fujiwara, K., Yamanaka, T., Nishida J., Matsunaga, S., Yano, H., and Mori, O.: Dynamics Behavior of Tether during Corer Projection for Tether Sampling, Proceedings of $16^{\text {th }}$ Space Engineering Conference, 2007, pp.29-34. (in Japanese)

8) Watanabe, T., Takikawa, H., Yukizane, M., Nakafushi, S., Mazawa,T., Ito, K., and Fujii, A. H.: Parabolic Flight Experiments for 3 dimensional Analysis of the Tethered Rigid Body, ISPS 2007, NARA, 2007

9) Heijning, S.P.A van de, Zandbergen, B.T.C.: Design of an electro-dynamic tape-tether deployment system, IAC-05-C4.6.02, 56th International Astronautical Congress, 2005.

10) Heijning, S.P.A van de, Zandbergen, B.T.C.: Testing of a tether deployment system using a cold gas thruster, IAC-05-D4.3.10, 56th International Astronautical Congress, 2005.

11) Takehara, S., Nohmi, M., Terumichi, Y., amd Sogabe, K.: Experimental Wxamination of the Motion of a Tethered System with Large Deformation and Large Displacement, Journal of Environment and Engineering, 2 (2007), pp. 64-75.

12) Watanabe, T., Fujii, A. H., and Kojima, H.: Quick Deployment of Bare Tape Tether and Overview of the Sounding Rocket Experiment, Proceedings of the AAS/AIAA Astrodynamics Specialists Conference, Mackinac Island, Michigan, USA, 19-23 August 2007, AAS 07-370. 